Errata Corrige

\title{
Modulation of the Redox State of the Copper Sites of Human Ceruloplasmin by Chloride
}

Giovanni Musci, Maria Carmela Bonaccorsi di Patti, and Lilia Calabrese

The above article appeared in Journal of Protein Chemistry, Vol. 14, No. 7, 1995, pp. 611-619. The acknowledgments section should correctly read:

\section{ACKNOWLEDGMENTS}

This work was partially supported by Consiglio Nazionale delle Ricerche (CNR) Progetto Finalizzato Invecchiamento code no. INV951590 and by MURST Fondi $40 \%$. 